\title{
REVEALING INSIGHTS OF USERS' PERCEPTIONS: AN APPROACH TO EVALUATE WEARABLE PRODUCTS BASED ON EMOTIONS
}

\author{
Liao, Ting; Tanner, Kesler; MacDonald, Erin \\ Stanford University
}

\begin{abstract}
The wearable products market is growing rapidly. Engaging users on an emotional level may be the key to long-term use and attracting new customers. While researchers have proposed various design approaches to realize design qualities for wearable devices, emotional needs are overlooked in the design process. To bridge this gap, we developed an approach with an online tool that uses a two-axis interactive collage for users to compare and evaluate wearable products with targeted emotion-related descriptive words. This approach enabled us to explore how users perceive products and identify types of emotions that were associated with their preferences for and perceptions of the product's form and visible characteristics. The study demonstrated this design approach to reveal insights into the relationships between product characteristics and design goals, such as user comfort, user delight, and perceived product usefulness. The results showed that products that resemble clothes were perceived as more delightful and comfortable. This study suggests that the approach can be further used to explore other design concepts or goals.
\end{abstract}

Keywords: User perception, Wearable devices, Emotional design, Design methods, User centred design

Contact:

Liao, Ting

Stanford University

Mechanical Engineering

United States of America

tingliao@stanford.edu

Cite this article: Liao, T., Tanner, K., MacDonald, E. (2019) 'Revealing Insights of Users' Perceptions: An Approach to Evaluate Wearable Products Based on Emotions', in Proceedings of the 22nd International Conference on Engineering Design (ICED19), Delft, The Netherlands, 5-8 August 2019. DOI:10.1017/dsi.2019.404 


\section{INTRODUCTION}

Tech-enabled wearable products have experienced increased use in recent years and have the potential to improve life in a variety of dimensions. In the last few years, some major brands, such as Apple, Sony, and Samsung, have introduced new sensors and communication technologies (e.g. Apple Watch, Sony's SmartWear, Samsung Gear, etc.). However, these competing products have very similar specifications, in terms of functions and features. Designers find it challenging to differentiate a product in such a space. Creating a specific emotional connection with customers may drive product adoption, retention, and continued use.

Evoking the emotion of delight shows promise in achieving such a connection, especially for a simple function such as monitoring task performance (e.g. a step counter). Hassenzahl (2004) found that the satisfaction level of the design for a MP3 player skin depended not only on the perceived usability but also on the hedonic attributes. The study by Bartl et al. (2013) showed that customers experiencing delight exhibited higher levels of product loyalty and purchase intention. In addition to user delight, the feeling of comfort may play a role in establishing a positive emotional connection. The study by Cahour et al. (2002) argued that the acceptance of a tool depended on its performance as well as the emotional comfort that users would feel or perceive. Thus, creating delight and comfort for users can promote longterm customer relationships of a wearable product and potentially secure a competitive position in the market.

While it is possible to intuitively design a product to have an emotional connection, such as in industrial design approaches, a more systematic approach is needed to measure this emotional connection in some way. Section 2.1 discusses past attempts at measuring this connection and Section 2.2 describes some existing methods of designing wearable devices. In this study, we proposed an approach with an online tool, a two-axis interactive collage, to understand how users perceive various wearable products both cognitively and emotionally. We developed this collage method based on the work of Guyton, who demonstrated the collage activity as an effective approach to establish product semantics to inspire sustainability concerns (Guyton, 2006), and the addition of a connection to perceptions by She and MacDonald (2014), who used it to trigger the five senses in designers. We created an experiment in which participants evaluated various wearable products under the target emotion-related or perceptionrelated criteria. Within the same interface, participants reported their perception, preference, and related emotions of the products in an integrated manner. Though emotions can be further evoked with physical interaction with products, this project focuses on the visual perception of form, functions, and features through a static online photographic representation, which usually happens as an exploratory phase in online shopping or before visiting a retail store.

Using this collage approach, we tested to understand if wearables that were positioned by participants as more closely resembling clothing evoked more user comfort and user delight. This clothing metaphor was inspired by the work of Dunne and Smyth (2007) about the device wearability (see Section 2.2 for details). Wearing clothes can involve both physical and social comfort and we tested to see if users transferred the feeling of being comfortable and delightful from wearing clothes to wearing electronic devices. We also analysed how participants associated emotion-related descriptive words with perceived user delight, user comfort, and product usefulness as well as user preference.

\section{BACKGROUND}

\subsection{Emotional design}

The value of meeting basic task-related needs has decreased. Kano developed a model in 1984, which categorizes product features into must-be, the-more-the-better, and delighting (Kano et al., 1984; Berger, 1993). MacDonald et al. (2006) further explored the feature classification methods of the Kano model and categorized the delighting features as the product attributes on which users received extra usefulness and satisfaction. Ealey and Troyano-Bermudez (1996) found it harder to predict customers' preferences when the automobile market was full of similar vehicles that customers could barely distinguish one from another, suggesting that a vehicle that created "delight and surprise" could potentially overcome this (Ealey and Troyano-Bermudez, 1996). A study by Bauer et al. (2006) demonstrated that customers reacted positively to emotional service aspects during online shopping and the appeal of the website strongly related to the judgement of its functionality and usability. 
Emotional design is an active field of research and has many analysis-rich approaches within the context of user-centred design and user experience design. Since late 1900s, Nagamachi has advocated Kansei engineering approach to handle consumers' emotional requirements in various design domain (Nagamachi, 1995, 2002). The Kansei approach addresses three following issues: 1) how to capture consumers' emotions to identify design requirements; 2) how to build the relationship between products and the consumers' emotional needs; and 3) how to design products to better fulfil the emotional needs. Kansei researchers have proposed many methods to capture/document emotions, for example, card sorting, semantic differential, etc. The most validated Kansei approaches rely on a non-interactive process with single focal product and a selection of Kansei adjectives (descriptors of emotions) to represent emotions in psychological realm.

\subsection{Wearable design}

One common approach to designing wearables is to realize a set of design qualities and to understand how the qualities shape user experience. Schirra and Bentley (2015) interviewed five participants who used a smartwatch for at least four months about the factors of purchase decisions and issues related to everyday usage. They identified several design considerations, including aesthetic dissimilarity, causal or sporty form, and colour. Lyons (2015) conducted a similar study with 50 respondents to understand factors that influenced buying and using a smartwatch, finding that style (formal/casual/sporty), similarity to a regular wristwatch, and size were the most important. Lyons also found that colour was one of the decisive design qualities for choosing a watch, and that sleek and simple watches were preferred over flashy ones. Maier et al. (2015) developed low-fidelity prototypes based on the existing smartphone technology after discussions with the focus group and tested the wearable technology acceptance to support the elderly. Dunne and Smyth (2007) conducted a study to investigate how sensory stimuli generated by wearing a device affected its wearability in terms of comfort and cognitive attention. They summarized physical, mental, emotional and social comfort as wearability and explored elements of clothing comfort, such as pressure, texture, moisture, etc. They concluded that wearability yielded conscious consequences and was essential for uses to accept the wearable devices. Yet, this study didn't involve an experiment or specific features for design.

Wearable research does not focus on the emotional connection as a primary design goal for wearable devices. For example, emotion is not mentioned in the study by Maier et al. (2015) about assistive technology for the elderly, in which positive feeling could be a key factor in adoption. Additionally, most studies do not involve a comprehensive representation of the alternative products/designs, but rather focus on exploring single product configuration/design. Reflecting on design qualities without being exposed to other available options causes evaluation in a vacuum, which is unrealistic given the large number of wearables.

\subsection{Collage tool design and coding}

Our method uses a two-axis collage tool, which was specifically developed from the work of Guyton, who demonstrated the collage activity as an effective approach to establish product semantics for sustainable products and to inspire sustainability concerns (Guyton, 2006).

In the collage activity, participants express their preference for various products by creating a two-axis map. First, they arrange product images on a grid with a set of labelled axes drawn, as illustrated in Figure 1(a). The axes are labelled at their extremities with words that are emotional or descriptive, such as "Like/Dislike" or "Comfortable/Not comfortable." Second, participants describe their selections by choosing words from a given list for each product image. This activity allows participants to evaluate multiple products holistically under various criteria, with different combinations of axes.

The authors have previously used this collage approach to prime designers into particular mindsets and to help them brainstorm design features that communicate sustainability to the customers (She and MacDonald, 2014). A follow-up study validated that a physical version of the collage-where participants physically handled product images to create a paper picture collage - enhanced students' design abilities to generate more effective features in terms of the environmental friendliness as judged by novice users (Liao and MacDonald, 2018).

We coded the two-axis interactive collage tool using Google's Firebase, as shown, in-use, in Figure 1(b). This tool allows participants to drag and drop products on the grid and click on the drop-down menu to choose descriptive words. A real-time database records the grid $\mathrm{x}$ and $\mathrm{y}$ positions of the products and the words selected. The link to this tool is https://product-drag-drop.firebaseapp.com. 
(a)

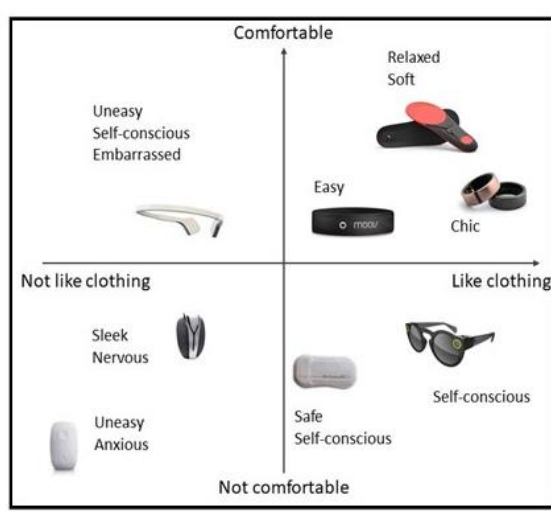

(b)

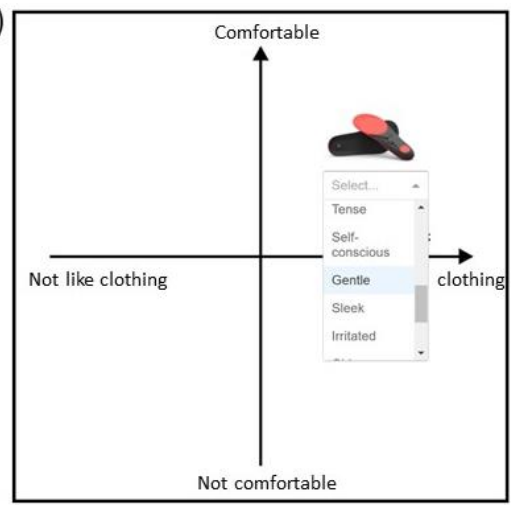

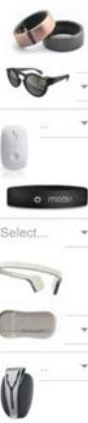

Figure 1. (a) Output of collage activity, (b) online tool in-use

\section{PROPOSITIONS}

We applied the two-axis interactive collage tool to test the following propositions:

Proposition 1: Wearable devices that resemble daily apparel or accessories are perceived as more comfortable. Being comfortable means physical ease and relaxation. Looking like apparel or clothes can remind users of the experience of wearing clothes and evoke the feeling of being comfortable.

Proposition 2: Wearable devices that resemble daily apparel or accessories are perceived as more delightful. Having users feel comfortable and relaxed can potentially let them reduce anxiety and experience delight. A low-tech or low-key aesthetic is expected to positively influence users' acceptance or emotions.

Proposition 3: Users perceive wearable devices that they like as more useful. Besides user comfort and user delight, the perceived usefulness (functionality) and users' preference are considered important factors for purchase intention and product retention, so are well worth exploring.

\section{COLlage eVAlUAtion APPROACH}

Derived from the physical collage activity explained in Section 2.3, we implemented a new experimental tool for users to evaluate various products and to express their preference. The details of the interactive collage activity are further described in the following sections.

\subsection{Selecting stimuli products for collage activity}

To select images for the collage, we benchmarked wearable devices available for purchase and selected eight products for the collage that either resemble apparel or have a novel look, and also have various methods of attachment/wear, see Table 1. Additionally, the products exist in the market yet are not as prevalent as smart watches or fitness wristbands, so we expect users' previous experiences will not bias the evaluations. Before the collage activity, we asked participants to review all the products with schematics of how they were attached to human body as well as brief descriptions of their functions, summarized in Table 1. The online experiment introduced each product in Table 1 on a separate screen in a randomized order.

Table 1. Pictures, schematics, and descriptions of the products for collage activity

\begin{tabular}{|l|l|}
\hline Product picture & Schematic \\
\hline & $\begin{array}{l}\text { This device is worn as a headband. It monitors heart rate } \\
\text { during workouts. It also gives exercise coaching. }\end{array}$ \\
\hline & $\begin{array}{l}\text { This is a shoe insole. It can function as a navigation device } \\
\text { for the blind individuals or as a fitness tracking system-for } \\
\text { example, counting steps taken, calories burned, and } \\
\text { monitoring and remembering workout routines. }\end{array}$ \\
\hline & $\begin{array}{l}\text { This device is worn as a ring on the finger. It has a 3-axis } \\
\text { accelerometer to detect motion, and an optical heart rate } \\
\text { sensor to track heart rate, active minutes, and calories } \\
\text { burned. }\end{array}$ \\
\hline
\end{tabular}




\begin{tabular}{|l|l|}
\hline & $\begin{array}{l}\text { This device is worn as a pair of sunglasses. It records a 10- } \\
\text { second video from user's perspective and syncs to } \\
\text { Snapchat, a messaging app for phones. }\end{array}$ \\
$\begin{array}{l}\text { This device is worn as a headband. It tracks mental activity } \\
\text { that helps reduce stress and settle the mind. The device uses } \\
7 \text { sensors that are applied to the occipital lobes. }\end{array}$ \\
$\begin{array}{l}\text { This device clips on belt, pocket, bra, etc. It tracks activity, } \\
\text { steps, and calories in order to monitor health. It helps to } \\
\text { understand what, where, and who causes you to be stressed, } \\
\text { calm, or productive. }\end{array}$ \\
$\begin{array}{l}\text { This device attaches to the upper back via a reusable } \\
\text { silicone adhesive. It easily fixes the posture by } \\
\text { strengthening the core back muscles and raising awareness. } \\
\text { It provides personalized training program and real-time } \\
\text { posture feedback via the mobile phone. }\end{array}$ \\
$\begin{array}{l}\text { This device attaches to the skin via an adhesive. It is a } \\
\text { body-worn sensor that naturally conforms to the contours of } \\
\text { the human body. It gathers raw kinematics and } \\
\text { electrophysiological data to monitor the wellbeing of the } \\
\text { patient after medical treatment. }\end{array}$ \\
\hline
\end{tabular}

\subsection{Axis labels}

The axis labels were determined regarding the propositions at hand (Section 3). Vertical-axis directly related to the targeted perceptions and emotions of each proposition (Table 2), and we used the same horizontal axis labels ("Not like clothing/Like clothing") so that participants could cognitively load as much as they wanted (e.g. opinion, features, etc.) on it.

Table 2. Axis labels for collage activity

\begin{tabular}{|c|c|c|c|}
\hline & Proposition 1 & Proposition 2 & Proposition 3 \\
\hline Horizontal axis label & $\begin{array}{c}\text { Not like clothing/ } \\
\text { Like clothing }\end{array}$ & $\begin{array}{c}\text { Not like clothing/ } \\
\text { Like clothing }\end{array}$ & Dislike/Like \\
\hline Vertical axis label & $\begin{array}{c}\text { Not comfortable/ } \\
\text { Comfortable }\end{array}$ & $\begin{array}{c}\text { Not delightful/ } \\
\text { Delightful }\end{array}$ & Not useful/Useful \\
\hline
\end{tabular}

\subsection{Descriptive words}

The words in the collage activity describe basic emotions, perceptions, and design characteristics. For emotional words, we retrieved terms both from the founding research in psychology that described primary and secondary emotions (Plutchik, 1984; Shaver et al., 1987; Smith, 2016) and from the manuals in psychiatric practice and personality tests (Hoffman Institute Foundation, 2015) to make sure words people use daily to self-report feelings were included. We also incorporated aesthetic words from visual design (Augustin et al., 2012), and perception-related words from a study of sustainable design (She and MacDonald, 2014). This collection of 473 words was decreased to 40 identifying words that relate to the collages' vertical axis labels, see Table 3, by computing the vector distances between the axis labels and the words using Global Vectors for Word Representation (GloVe) algorithm (Pennington et al., 2014). GloVe represents each word as a vector and is trained on aggregated global word-word cooccurrence statistics from a corpus.

Table 3. Lists of descriptive words

\begin{tabular}{|c|c|c|c|}
\hline Comfortable & Not comfortable & Delightful & Not delightful \\
\hline Relaxed & Awkward & Delicious & Uncomfortable \\
Quiet & Unpleasant & Joyful & Disgusting \\
Soft & Uneasy & Admirable & Harsh \\
Sleek & Self-conscious & Blissful & Bitter \\
Gentle & Embarrassed & Joyous & Miserable \\
\hline
\end{tabular}




\begin{tabular}{|c|c|c|c|}
\hline Safe & Irritated & Playful & Dreadful \\
Calm & Anxious & Sweet & Full \\
Chic & Tense & Ecstatic & Hateful \\
Easy & Scared & Festive & Awful \\
Secure & Nervous & Eager & Fearful \\
\hline
\end{tabular}

\section{EXPERIMENT PROCEDURE}

Four-hundred participants were recruited on Amazon Mechanical Turk (AMT). Criteria for selecting participants followed the recommendations by Mason and Suri (2012) and included physically locating at the United States and a greater than 60\% approval rate for previous AMT tasks. The experimental procedure is show in Figure 2. The participants were first introduced to the focal products with the product pictures, schematics, and brief descriptions about how the products were attached and functioned. The products were presented in sequence in a randomized order. The full list of products is in Table 1. Second, they received a tutorial on the collage tool. Then, the participants performed the collage activity. Finally, they answered questions on their wearable use and purchases.

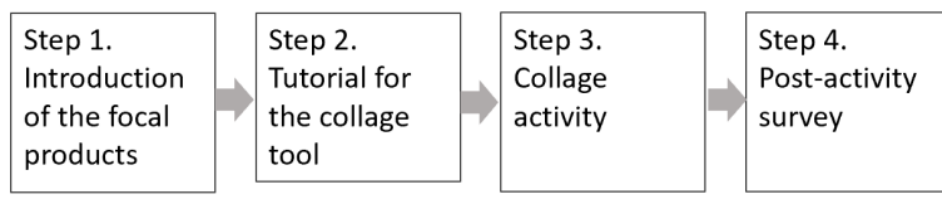

Figure 2. Overview of experiment procedure

\section{DATA AND ANALYSIS}

We collected 351 valid responses, as determined by response time, which was required to be within one standard deviation of the mean response time of 7.0 minutes. $44 \%$ of the participants are female and 54\% are male. $43 \%$ of them have ages between $26-34$ and $36 \%$ have ages between $35-54.63 \%$ of participants reported previously using or buying a wearable product, and the most popular choice was Fitbit. Regardless of whether or not they currently own a wearable device, $43 \%$ of participants reported considering buying a wearable device.

The final positions of products were recorded as well as the descriptive words. The average $\mathrm{x}$ and $\mathrm{y}$ positions were calculated to represent aggregated rating results of products by 351 judges. The boundary of the collage is from -300 to 300 (figures for the average positions represent the zoomed in views). The numeric values indicate relative positions of the products on each category but do not have specific meanings.

\subsection{Evaluating user comfort}

Figure 3 shows average $\mathrm{x}$ - and $\mathrm{y}$-values for each product for axes "Not comfortable/Comfortable" and "Not like clothing/Like clothing". The positive linear trend $\left(\mathrm{R}^{2}=0.79\right)$ indicates that wearables that are more like clothing are positioned on the grid as more comfortable.

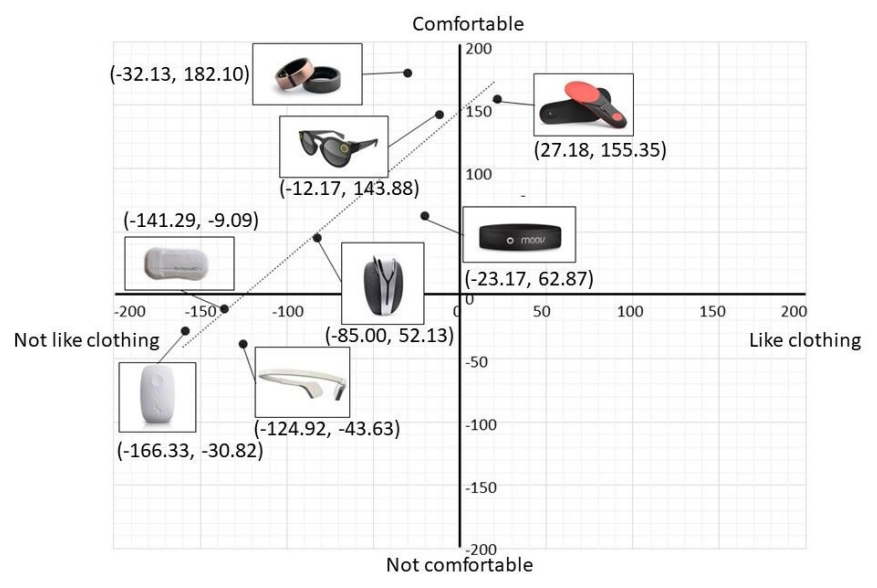

Figure 3. Average positions for axis "Not comfortable/Comfortable" 


\subsection{Evaluating user delight}

Figure 4 shows average $\mathrm{x}$ - and y-values for each product for axes "Not delightful/Delightful" and "Not like clothing/Like clothing." The positive linear trend $\left(\mathrm{R}^{2}=0.76\right)$ indicates that wearables that are more like clothing are positioned as more delightful.

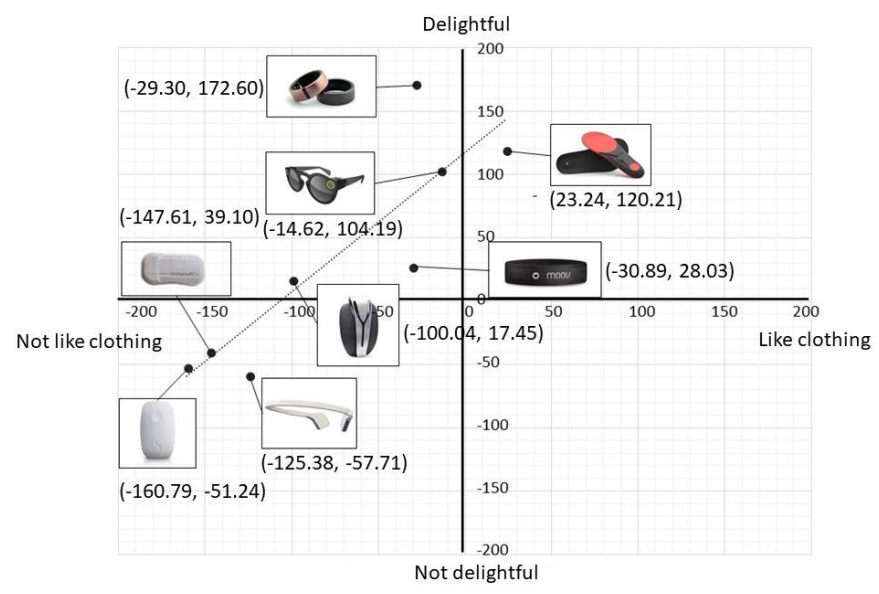

Figure 4. Average positions for axis "Not delightful/Delightful"

\subsection{Evaluating product usefulness}

Figure 5 shows average $\mathrm{x}$ - and $\mathrm{y}$-values for each product for axes "Not useful/Useful" and "Dislike/Like". The positive linear trend $\left(\mathrm{R}^{2}=0.67\right)$ indicates that wearables that are more liked are positioned as more useful. Note that the scale is different from that of Figure 3 and Figure 4, as most products locate in the second quadrant, indicating most participants considered these products useful but did not like them.

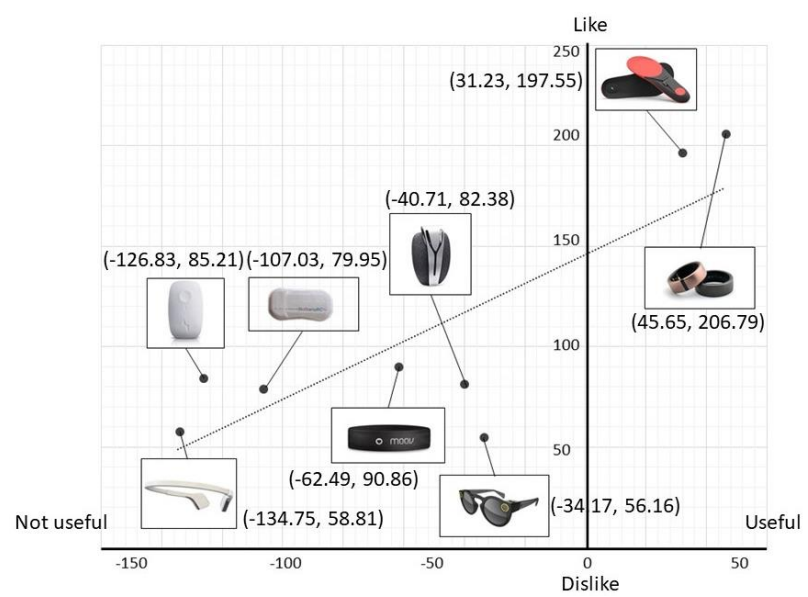

Figure 5. Average positions for axis "Not useful/Useful"

\subsection{Choosing descriptive words}

As described in Section 4.3, in the collage tool, participants arranged product pictures and identified related descriptive, emotion- and perception-words from a given list. The positions of the descriptive words were recorded as the positions of the associated product pictures. 

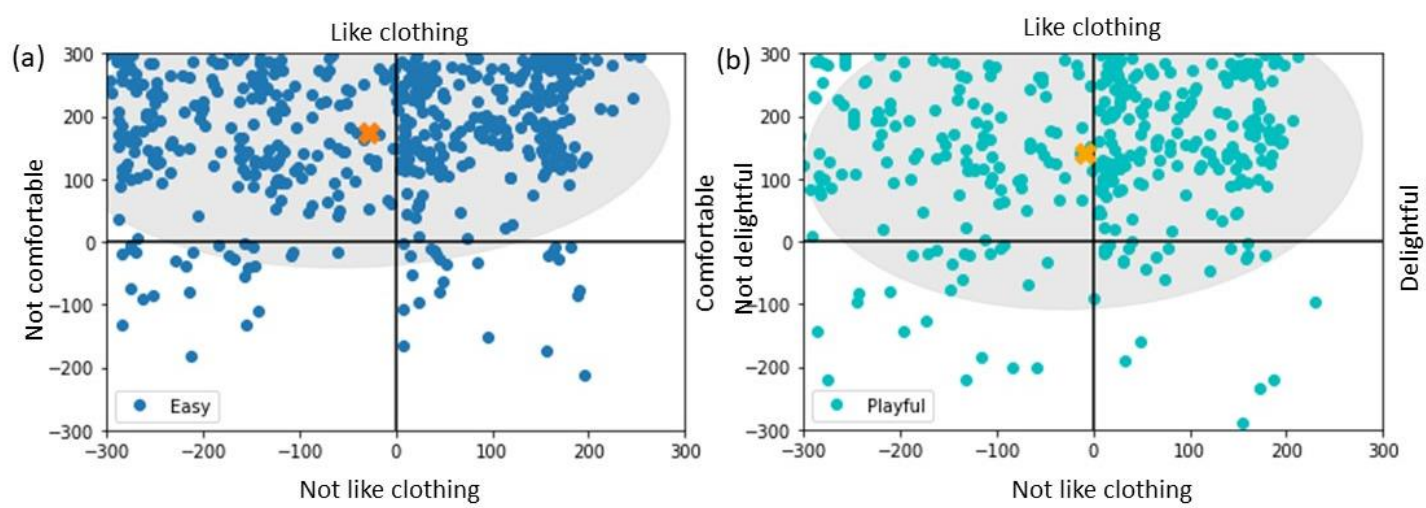

Figure 6. Positions of the descriptive word (a) "Easy" on the collage with axis labels "Not comfortable/Comfortable" and (b) "Playful" with axis labels "Not delightful/Delightful"

In the collage with axis labels "Not comfortable/Comfortable" and "Not like clothing/Like clothing", each word was selected 233.9 times on average with a standard deviation (SD) of 141.1. The top five most frequently selected words include "Easy", "Awkward", "Secure", "Sleek" and "Unpleasant". Shown above (blue dots) in Figure 6(a) are the positions of the most frequently selected word "Easy", which was chosen 540 times for various products in this collage. Its average position $(-27.72,174.8)$ is shown as the orange " $X$ ". The light grey ellipse indicates the confidence region with two standard deviations from the average position along both axes. The region almost equally expands the first and second quadrants on the plot. This plot indicates that the ease to use or wear remains as a critical characteristic, especially for products that provide intimate body contact like wearable devices.

In this collage with axis labels "Not delightful/Delightful" and "Not like clothing/Like clothing", each word was selected 176.6 times on average with a SD of 153.1. The words being most selected are "Uncomfortable", "Playful", "Admirable", "Eager", and "Dreadful". Figure 6(b) shows the positions of word "Playful" in the collage, which was selected 372 times in total, as the most frequently used positive word in this criterion. The average position locates closely to the $y$-axis, which indicates that being playful is not related to if the device looks like clothing or not.
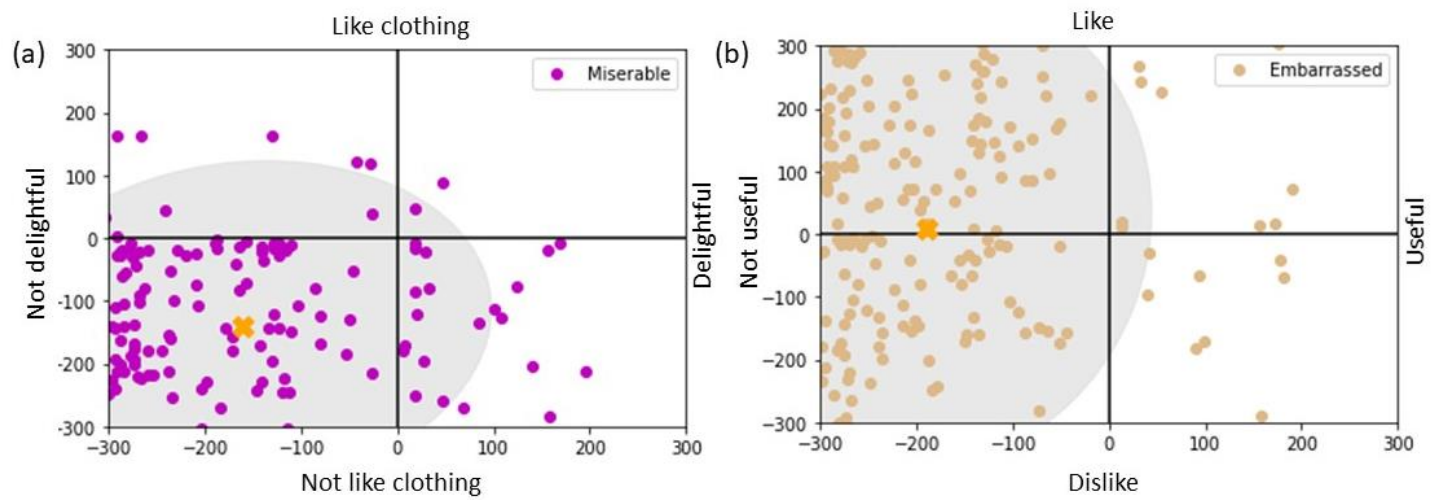

Figure 7. Positions of the descriptive word (a) "Miserable" on the collage with axis label "Not delightful/Delightful" and (b) "Embarrassed" with axis label "Not useful/Useful"

In addition to desired qualities, the word "Miserable" has the minimum scores on both axes on the collage with axis labels "Not delightful/Delightful" and "Not like clothing/Like clothing", and the points clearly cluster in the lower left quadrant in Figure 7(a). This suggests that while being alike clothes may not cause a more delightful experience, being unlike clothes or obtrusive can be associated with the undesirable feeling.

Figure 7(b) shows the positions of the word "Embarrassed" in the collage with axis labels "Not useful/Useful" and "Dislike/Like". This word has the minimum average score on the scale of product usefulness. Most words locate to the left of the axis "Dislike/Like" but are spread out along the axis "Not useful/Useful". This indicates that the perceived usefulness of a product is not relevant to users' feeling of embarrassment. 


\section{DISCUSSION AND CONCLUSION}

In this paper, we demonstrated the design and development of a new approach that allows users to interactively evaluate multiple products, under various targeted criteria and to self-reflect on their emotions when perceiving the products. As for Proposition 1 and 2, the user responses suggest that the wearable products more closely resembling clothing are perceived as both more comfortable and more delightful, which support the propositions. In addition, the outputs indicate that products users like more are considered as more useful; however, 6 out of 8 products received negative average scores on the scale of liking. These results support Proposition 3 and demonstrate the gap between fulfilling the functional requirements and providing user satisfaction, as suggested by previous literature. The study underscores the need for further product development on emotional needs.

This approach also facilitates identifying descriptive words regarding perception, emotions and aesthetics of each product comparatively under various criteria. The word "Easy" was the most frequently chosen when users evaluated products' comfort. The word "Playful" being the most frequently selected positive word to reflect user delight uncovers the design opportunity to make products fun to play with. Using "Easy" and "Playful" as design criteria, we created a use case of the wearable devices for monitoring infants. The prototype incorporates a playful appearance with printed cartoon patterns and flexible sensors to evoke an easy and playful experience. We will validate this approach by testing if the prototype can result in user perception of a more useful, delightful, and useful product.

The study demonstrates this two-axis interactive collage tool to investigate relationships between product characteristics and emotional needs, as well as to identify meaningful design words. It focuses on revealing insights from users' visual perception, with a context of product evaluation, instead of from a text-based emotion assessment. This approach simulates a realistic shopping situation, where potential customers evaluate available options in a similar manner. Furthermore, the axes and word banks can be manipulated to explore different design dimensions, as well as the online feature makes it accessible to a larger and more diverse population.

\section{LIMITATIONS AND FUTURE WORK}

There are many other characteristics that relate to user comfort and delight. As this study only focuses on visual perception of the product images, other attributes that relate to material functionality were not included. Some design attributes, such as surface finish and colour, that were found to have a significant impact on the purchase intension can be included in the future experiment to provide an enriched design guideline. This study aims to mimic the early-stage exploration in online shopping, and an in-person experiment can be conducted to understand user interactions with the tactile features. The selection of the product images can potentially influence the outcome. The focal products in this study varied in terms of functions and outlooks and expanded the design space as much as possible. This variety can potentially make it difficult for users to compare. The number of wearable products being presented was relatively small, probably unable to capture many sectors in wearable design; however, presenting more representative products might increase task difficulty or cause mental burden for participants.

This study only included an online (as opposed to in-person) collage activity so it could be more accessible to the general population. We do not anticipate any difference of the results caused by the modes of the experiment, but any technical difficulty with internet or computer can distort the results. In addition, the axes corresponding to the three propositions were presented in the same order to all participants, which could have potentially created fixation.

Regarding the hypotheses at hand, we only tested linear relationships within individual pairs of axes. Furthermore, participants were surveyed about their experience using wearable devices and future purchase intention. These factors can potentially influence participants' expectations and evaluations of the products, yet they are not taken into analysis for this study.

This approach enables identifying design words that are associated with user-perceived comfort, delight and usefulness. The next step will be to transfer these design words into guidelines for industrial designers. More testing and/or industrial designers with related training are needed to embody the words in new product designs. 


\section{REFERENCES}

Augustin, M.D., Carbon, C.C. and Wagemans, J. (2012), “Artful terms: A study on aesthetic word usage for visual art versus film and music", $i$-Perception, Vol. 3 No. 5, pp. 319-337.

Bartl, C., Gouthier, M.H. and Lenker, M. (2013), "Delighting consumers click by click: Antecedents and effects of delight online”, Journal of Service Research, Vol. 16 No. 3, pp. 386-399.

Bauer, H.H., Falk, T. and Hammerschmidt, M. (2006), "eTransQual: A transaction process-based approach for capturing service quality in online shopping”, Journal of Business Research, Vol. 59 No. 7, pp. 866-875.

Berger, C. (1993), "Kano's methods for understanding customer-defined quality”, Center for Quality Management Journal, Vol. 2 No. 4, pp. 3-36.

Cahour, B., Lorant, F. and Sanchiz, F. (2002), "Use of teleconferencing: perturbing cognitive and social comfort", Proceedings of the 11th European Congress of Cognitive Ergonomics (Cognition, Culture and Design), pp. 7-10.

Dunne, L.E. and Smyth, B. (2007), “April. Psychophysical elements of wearability”, Proceedings of the SIGCHI Conference on Human Factors in Computing Systems, ACM, pp. 299-302.

Ealey, L. and Troyano-Bermúdez, L. (1996), “Are automobiles the next commodity?”, The McKinsey Quarterly No. 4, pp. 62.

Guyton, A.A. (2006), Developing sustainable product semantics for consumer products: A sustainable designer's guide, Doctoral dissertation, Georgia Institute of Technology.

Hassenzahl, M. (2004), “The interplay of beauty, goodness, and usability in interactive products”, Humancomputer Interaction, Vol. 19 No. 4, pp. 319-349.

Kano, N., Seraku, N., Takahashi, F. and Tsuji, S. (1984), “Attractive quality and must-be quality”, The Journal of the Japanese Society for Quality Control, Vol. 14 No. 2, pp. 39-48.

Kittur, A., Chi, E.H. and Suh, B. (2008), "Crowdsourcing user studies with Mechanical Turk", Proceedings of the SIGCHI conference on human factors in computing systems, pp. 453-456, ACM.

Liao, T. and MacDonald, E. (2018), "Effects of collage priming on sustainable design idea creation and assessment", Proceedings of the International Design and Engineering Technical Conference, pp. V007T06A056-V007T06A056.

Hoffman Institute Foundation (2015), Feeling list. Available at: https://www.hoffmaninstitute.org/wpcontent/uploads/Practices-FeelingsSensations.pdf (Accessed Dec 15th, 2018).

Lyons, K. (2015), "What can a dumb watch teach a smartwatch: informing the design of smartwatches", Proceedings of the 2015 ACM International Symposium on Wearable Computers (ISWC '15), pp. 3-10. https://doi.org/10.1145/2802083.2802084.

MacDonald, E., Backsell, M., Gonzalez, R. and Papalambros, P. (2006), “The Kano method's imperfections, and implications in product decision theory", Proceedings of the 2006 International Design Research Symposium.

Maier, A.M., Özkil, A.G., Bang, M.M. and Forchhammer, B.H. (2015), "Remember to remember: a feasibility study adapting wearable technology to the needs of people aged 65 and older with mild cognitive impairment (MCI) and Alzheimer's Dementia”, Proceedings of International Conference on Engineering Design (ICED), Vol. 1, pp. 331-340.

Mason, W. and Suri, S. (2012), "Conducting behavioral research on Amazon's Mechanical Turk", Behavior Research Methods, Vol. 44 No. 1, pp. 1-23.

Nagamachi, M. (1995), "Kansei engineering: a new ergonomic consumer-oriented technology for product development”, International Journal of Industrial Ergonomics, Vol. 15, pp. 3-11.

Nagamachi, M. (2002), ""Kansei engineering as a powerful consumer-oriented technology for product development"', Applied Ergonomics No. 33, pp. 289-294.

Pennington, J., Socher, R. and Manning, C. (2014), "GloVe: global vectors for word representation”, Proceedings of the 2014 Conference on Empirical Methods in Natural Language Processing, pp. 1532-1543.

Plutchik, R. (1984), “Emotions: A general psychoevolutionary theory”, Approaches to Emotion, pp. 197-219.

Shaver, P., Schwartz, J., Kirson, D. and O'connor, C. (1987), "Emotion knowledge: Further exploration of a prototype approach", Journal of Personality and Social Psychology, Vol. 52 No. 6, pp. 1061.

Schirra, S. and Bentley, F.R. (2015), "It's kind of like an extra screen for my phone: Understanding Everyday Uses of Consumer Smart Watches", Proceedings of the 33rd Annual ACM Conference Extended Abstracts on Human Factors in Computing Systems, ACM, pp. 2151-2156.

She, J. and MacDonald, J. (2014), "Priming designers to communicate sustainability", Journal of Mechanical Design, Vol. 136 No. 1, pp. 011001.

Smith, T.W. (2015), “The Book of Human Emotions: An Encyclopedia of Feeling from Anger to Wanderlust”, Profile Books. 